\title{
Impacto da queima da cana-de-açúcar sobre internações hospitalares por doenças respiratórias
}

\author{
The impact of sugarcane burning on hospitalization due to \\ respiratory diseases
}

Dionei Ramos (https://orcid.org/0000-0002-2956-7399) ${ }^{1}$

Paula Roberta Silva Pestana (https://orcid.org/0000-0001-9312-8713) ${ }^{1}$

Iara Buriola Trevisan (https://orcid.org/0000-0003-0743-3231) ${ }^{1}$

Diego Giulliano Destro Christofaro (https://orcid.org/0000-0001-9917-9992) ${ }^{2}$

Guilherme Yassuyuki Tacao (https://orcid.org/0000-0002-7500-0772) ${ }^{1}$

Iris Cristina Coripio (https://orcid.org/0000-0002-4312-0788) ${ }^{1}$

Aline Duarte Ferreira (https://orcid.org/0000-0003-0102-1738) ${ }^{1}$

Ercy Mara Cipulo Ramos (https://orcid.org/0000-0002-3310-7336) ${ }^{1}$
${ }^{1}$ Departamento de Fisioterapia, Faculdade de Ciência e Tecnologia, Universidade Estadual Paulista (UNESP). R. Roberto Simonsen 305, Departamento de Fisioterapia. 19060-900 Presidente Prudente SP Brasil.

dionei-ramos@bol.com.br ${ }^{2}$ Departamento de Educação Física, Faculdade de Ciência e Tecnologia, UNESP. Presidente Prudente SP Brasil.

\begin{abstract}
The scope of this article is to evaluate the impact of emissions from sugarcane burning on hospital admission numbers for respiratory diseases in a sugarcane region. Hospital admission records for respiratory diseases were acquired from the database of the Hospital Information System of the Unified Health System (SIH-SUS) in the period from March 28, 2011 to December 28, 2012. Levels of PM10, NO2, O3, Temperature and Relative Humidity were recorded. Logistic regression models were created to analyze the association between the total number of hospitalizations, atmospheric pollutants and meteorological variables. A total of 1,179 hospitalization admissions were recorded, with a significant increase in cases of pneumonia in the burning period ( $p=$ 0.005). Likewise, it was observed that the cluster of PM10 and NO2 was influenced 67.9\% (95\% CI: 11.111-2.537) followed by cluster PM10, NO2, O3 and Temperature that influenced $91.1 \%$ (95\% CI: 1.116; 3.271) in the total number of hospitalization admissions. During the sugarcane burning period there were more hospitalization admissions due to respiratory tract diseases, mainly pneumonia, where the influence of air pollutants and temperature in the process of illness in the population was detected.
\end{abstract}

Key words Air pollutants, Sugarcane, Respiratory tract diseases, Pneumonia, Meteorological variables
Resumo O objetivo deste artigo é avaliar o impacto das emissões da queima da cana-de-açúcar sobre o número de internações hospitalares por doenças respiratórias em uma região canavieira. Registros de internações hospitalares por doenças respiratórias foram adquiridas a partir da base de dados referentes ao sistema de informações hospitalares do sistema único de saúde (SIH-SUS), no período de 28 de março de 2011 a 28 de dezembro de 2012. Níveis de $\mathrm{MP}_{10}, \mathrm{NO}_{2}, \mathrm{O}_{3}$, temperatura e umidade relativa foram registradas. Modelos de regressão logística foram construídos para analisar a associação entre o número total de internações, poluentes atmosféricos e variáveis meteorológicas. Foram registradas 1.179 internações, dentre elas a pneumonia mostrou aumento significativo no período de queima $(p=0,005)$. Neste mesmo período observou-se que o cluster $M P_{10}$ e $\mathrm{NO}_{2}$ influenciou 67,9\% (IC95\%: 11,1112,537), seguido do cluster $M P_{10}, N_{2}, \mathrm{O}_{3}$ e temperatura que influenciou 91,1\% (IC95\%: 1,116; 3,271) no número total de internações. Durante o periodo de queima houve maior número de internações por doenças respiratórias, principalmente de pneumonia, quando foi observada a influência dos poluentes e temperatura no processo de adoecimento da população.

Palavras-chave Poluentes atmosféricos, Cana-de -açúcar, Doenças respiratórias, Pneumonia, Modalidades meteorológicas 


\section{Introdução}

O impacto da poluição atmosférica sobre a saúde humana vem sendo tema de vários estudos ${ }^{1-4}$, evidenciando um grave problema de saúde pública. Em regiões canavieiras, por exemplo, a queima noturna da palha da cana-de-açúcar pode ser considerada como o maior fator contribuinte de agravos em cidades interioranas ${ }^{5,6}$. Além de afetarem as comunidades próximas, os poluentes atmosféricos podem viajar quilômetros e atingir locais distantes da fonte de emissão e, mesmo que abaixo dos níveis determinados pela legislação, são capazes de provocar efeitos à saúde da população $0^{7-11}$.

A nível mundial, 25\% das terras correspondentes a plantações de cana-de-açúcar estão localizadas no Brasil, o Estado de São Paulo é o maior produtor desta safra, crescendo em $121 \%$ a cada década. A queima da cana está diminuindo gradualmente a cada ano desde 2002, quando a lei de suspensão da queima entrou em vigor, tendo uma prospectiva gradual de mecanização de toda área do Estado de São Paulo, entretanto, sabe-se que essa queima da palha de cana-de-açúcar e sua consequente emissão de poluentes ainda vão impactar a saúde das regiões canavieiras não só do Estado de São Paulo, mas a nível País onde a queima da cana de açúcar ainda é um problema para a saúde humana ${ }^{12,13}$.

A queima da palha da cana-de-açúcar pode gerar uma grande quantidade de aerossóis, material particulado (MP), gases como monóxido de carbono (CO) e dióxido de carbono $\left(\mathrm{CO}_{2}\right)$, aldeídos, metano $\left(\mathrm{CH}_{4}\right)$, óxidos de nitrogênio (NOx) e outros hidrocarbonetos policíclicos aromáticos (PAHs). Uma vez na atmosfera, alguns desses gases como metano, dióxido de nitrogênio $\left(\mathrm{NO}_{2}\right)$ e hidrocarbonetos podem produzir poluentes secundários como o ozônio $\left(\mathrm{O}_{3}\right)$, por meio de uma reação fotoquímica ${ }^{14,15}$.

Os efeitos da exposição humana à estes poluentes são de caráter agudo ou crônico, e vão desde tosse seca e cansaço, irritação no nariz e garganta, chegando até a causar aumento de liberação de células inflamatórias e alterações no clearance mucociliar nasal ${ }^{16,17}$. Um estudo realizou uma projeção de mortalidade e internações hospitalares na rede pública de saúde no Estado de São Paulo, onde estimaram que entre 2011 e 2030, o total de mortes chegaria a mais de $246 \mathrm{mil}$ óbitos e 918 mil internações apenas por causas cardiovasculares, respiratórias e neoplasias em grupos mais susceptíveis ${ }^{18}$.
Segundo o mesmo autor, a mortalidade geral atribuída ao MP apresentou um aumento de $96 \%$ no período de análise, com 9.384 mortes em 2011 e uma projeção de 18.407 mortes para $2030^{18}$. Bayram ${ }^{19}$ realizou um estudo mostrando associação entre $\mathrm{NO}_{2}$ e $\mathrm{O}_{3}$, o qual constatou que havia ocorrido o aumento da permeabilidade do tecido brônquico e células inflamatórias em pacientes com doenças respiratórias crônicas, como asma e doença pulmonar obstrutiva crônica (DPOC). Diante da temática e das lacunas ainda a serem preenchidas na literatura científica, o presente estudo teve como objetivo avaliar o impacto das emissões da queima noturna da palha da canade-açúcar sobre o número de internações hospitalares por doenças respiratórias em uma região canavieira do estado de São Paulo.

\section{Métodos}

O estudo foi realizado na cidade de Presidente Prudente, São Paulo, Brasil, situada a 587 quilômetros da capital, com uma população de 223.749, de acordo com dados do Instituto Brasileiro de Geografia e Estatística (IBGE) ${ }^{20}$. Foram registrados número de internações hospitalares e emergenciais por doenças respiratórias (DR) a partir da base de dados referentes ao sistema de informações hospitalares do sistema único de saúde (SIH-SUS), disponibilizado pelo Hospital Regional de Presidente Prudente, de acordo com a Classificação Estatística Internacional de Doenças e Problemas Relacionados à Saúde - Décima Revisão (CID-10). Dentre as doenças, foram observadas internação por DPOC (CID-10: J44), asma (CID-10: J45), pneumonia (CID-10: J12-J18), broncopneumonia (CID-10: J18.0) e infecções de vias aéreas superiores (IVAS, CID10: J00-J06), no período de 28 de março de 2011 a 28 de dezembro de 2012 .

Foram incluídos somente os registros de indivíduos residentes na cidade de Presidente Prudente e quando o diagnóstico médico se encontrava bem definido para as DR. O estudo foi aprovado pelo Comitê de Ética em Pesquisa da Faculdade de Ciências e Tecnologia (FCT/ UNESP), Campus de Presidente Prudente-SP.

O material $\mathrm{MP}_{10}, \mathrm{NO}_{2}, \mathrm{O}_{3}$, temperatura $(\mathrm{T}) \mathrm{e}$ umidade relativa do ar (UR) foram obtidas por amostragem ativa através de uma rede automática de monitoramento do estado de São Paulo, localizada em Presidente Prudente/SP ${ }^{21}$. Esta rede registra as concentrações dos poluentes através 
do sistema de telemetria e são disponibilizados diariamente, apresentando um resumo das condições da poluição atmosférica e meteorológica das últimas 24 horas.

Para a análise estatística, foi utilizado o SPSS, versão 15.0. O teste de Mann-Whitney foi utilizado para verificar as diferenças entre as médias de variáveis contínuas, uma vez que estas não mostraram distribuição normal no teste de normalidade de Kolmogorov-Smirnov. Para a análise de regressão logística, informamos que a definição de cada categoria de doença foi estabelecida como nenhuma internação ou uma ou mais internações por dia.

Posteriormente, modelos de regressão logística foram construídos separadamente para analisar a associação entre a frequência do número total de internações por DR, poluentes atmosféricos e variáveis meteorológicas. Nos modelos, foram incluídos as variáveis de ajuste como $\mathrm{MP}_{10}$, $\mathrm{NO}_{2}, \mathrm{O}_{3}, \mathrm{~T}$ e UR, durante o período de queima da palha da cana-de-açúcar.

Para esta regressão foram elaborados clusters dos poluentes a partir do somatório de quantidade de $\mathrm{MP}_{10}, \mathrm{NO}_{2 \mathrm{e}} \mathrm{O}_{3}$. Para isso, todos os poluentes foram transformados em Score $Z$ (formula: (amostra - média) / DP) e os clusters foram feitos a partir da somatória entre as possíveis combinações. Os dados de T e UR foram combinados aos clusters que apresentaram significância afim de identificar a influência meteorológica junto aos poluentes sobre as internações por DR. O aumento do risco relativo foi calculado usando a formula $(\operatorname{Exp}(B)-1){ }^{\star} 100$. O nível de significância adotado considerado para todos os testes foi de $5 \%(p<0,05)$.

\section{Resultados}

Na Tabela 1 estão representadas as principais características do estudo. Foram registradas 1.179 internações por DR, com no mínimo zero de internações e máximo de oito internações por dia. As médias das concentrações de $\mathrm{MP}_{10}, \mathrm{NO}_{2}$ e $\mathrm{O}_{3}$ apresentaram-se dentro dos limites classificados como "Boa" de acordo com a OMS 22 (0-50 $\mu \mathrm{g} /$ $\mathrm{m}^{3}, 0-200 \mu \mathrm{g} / \mathrm{m}^{3}, 0-100 \mu \mathrm{g} / \mathrm{m}^{3}$, respectivamente).

Ao longo do estudo, quando estratificado pelo período de não queima (162 dias) e queima (350 dias) da cana-de-açúcar, houve diferença significativa do número de internações por DR no período da queima, em comparação ao período de não queima $(\mathrm{p}=0,011)$. No entanto, den- tre as doenças, apenas a pneumonia apresentou aumento significativo no número de internações no período de queima $(\mathrm{p}=0,005)$ (Tabela 2).

As concentrações de $\mathrm{MP}_{10}, \mathrm{NO}_{2}$ e $\mathrm{O}_{3}$ aumentaram significantemente durante o período de queima ( $\mathrm{p}<0,0001)$, em contrapartida os dados de T e UR diminuíram significantemente neste mesmo período ( $\mathrm{p}<0,0001$; Tabela 3 ). Houve correlação positiva entre $\mathrm{O}_{3}$ e $\mathrm{T}(\mathrm{r}=0,644$; $\mathrm{p}<$ 0,0001).

$\mathrm{Na}$ análise de regressão logística, pode-se observar que o número total de internações no período de queima da cana-de-açúcar sofreu influência de 67,9\% do $\mathrm{MP}_{10}$ e $\mathrm{NO}_{2}(\operatorname{Exp}(\mathrm{B}) 1,679$; IC95\%: 11,111-2,537). No entanto, a combinação de $\mathrm{MP}_{10}, \mathrm{NO}_{2}, \mathrm{O}_{3}$ e $\mathrm{T}$ influenciou $91,1 \%$ no número total de internações por DR no período de queima $(\operatorname{Exp}(B) 1,911$; IC95\%: 1,116; 3,271) (Tabela 4).

\section{Discussão}

O presente estudo teve como objetivo avaliar o impacto das emissões de poluentes por meio da queima da cana-de-açúcar sobre as internações hospitalares por DR em uma região canavieira. Dentre os principais achados, observou-se um aumento no número de internações por DR, principalmente por pneumonia, no período de queima de cana-de-açúcar. Além disso, o aumento dos poluentes associado com a diminuição da temperatura mostrou relação direta sobre o número total de internações por doenças respiratórias neste período.

A queima da cana-de-açúcar é responsável pela produção e emissão de MP e gases tóxicos, que possuem efeitos deletérios à saúde humana à curto e longo prazos ${ }^{23-25}$. Dentre esses poluentes, o MP apresenta maior toxidade $\mathrm{e}^{26} \mathrm{e}$, quando inalados, pode desencadear processos inflamatórios a partir da formação de agentes oxidantes intracelulares, produzindo mecanismos como o aumento da reatividade brônquica, redução do transporte mucociliar, estresse oxidativo, sibilância, infecções de vias aéreas e exacerbações de crises asmáticas e da DPOC $^{27-29}$.

Em um estudo realizado com MP, por Atkinson et al. ${ }^{23}$, relataram que este tipo de poluente pode provocar danos físicos ao sistema respiratório. Alguns outros estudos também têm sugerido que o MP está associado a toxidade respiratória em estudos in vivo, in vitro e epidemiológicos ${ }^{24-26}$. Além disso, outros estudos apontam que a expo- 
Tabela 1. Características das principais variáveis do estudo, Oeste do Estado de São Paulo, Brasil, 2011 a 2012.

\begin{tabular}{lccccccc}
\hline \multicolumn{1}{c}{ Variáveis } & Média & DP & Mínimo & Máximo & \multicolumn{3}{c}{ Percentils } \\
\cline { 6 - 8 } & & & & & 25th & 50th & 75th \\
\hline Total de internações $(\mathrm{n}=1.179)$ & 2,3 & 1,3 & 1 & 8 & 1,0 & 2,0 & 3,0 \\
DPOC $(\mathrm{n}=114)$ & 0,2 & 0,5 & 0 & 3 & 0,0 & 0,0 & 0,0 \\
Asma $(\mathrm{n}=35)$ & 0,1 & 0,3 & 0 & 2 & 0,0 & 0,0 & 0,0 \\
Pneumonia $(\mathrm{n}=497)$ & 1,0 & 1,0 & 0 & 7 & 0,0 & 1,0 & 1,0 \\
Broncopneumonia $(\mathrm{n}=338)$ & 0,7 & 0,9 & 0 & 4 & 0,0 & 0,0 & 1,0 \\
Infecções VAS $(\mathrm{n}=195)$ & 0,4 & 0,7 & 0 & 4 & 0,0 & 0,0 & 1,0 \\
Poluentes Atmosféricos & & & & & & & \\
$\quad \mathrm{MP}_{10}\left(\mu \mathrm{g} / \mathrm{m}^{3}\right)$ & 24,1 & 13,1 & 4,3 & 82,9 & 13,9 & 22,2 & 31,6 \\
$\mathrm{NO}_{2}\left(\mu \mathrm{g} / \mathrm{m}^{3}\right)$ & 16,2 & 7,3 & 3,7 & 43,1 & 11,2 & 14,6 & 21,0 \\
$\mathrm{O}_{3}\left(\mu \mathrm{g} / \mathrm{m}^{3}\right)$ & 48,3 & 16,3 & 10,0 & 95,0 & 37,2 & 47,1 & 58,3 \\
Variáveis Meteorológicas & & & & & & & \\
$\mathrm{T}\left({ }^{\circ} \mathrm{C}\right)$ & 23,7 & 3,8 & 9,6 & 32,8 & 21,6 & 24,2 & 26,4 \\
$\quad \mathrm{UR}(\%)$ & 61,8 & 14,4 & 24,3 & 98,5 & 52,0 & 61,8 & 71,5 \\
\hline
\end{tabular}

DP: desvio padrão; DPOC: doença pulmonar obstrutiva crônica; VAS: vias aéreas superiores; $\mathrm{MP}_{10}$ : material particulado de 10 micrômetros; $\mathrm{NO}_{2}$ : dióxido de nitrogênio; $\mathrm{O}_{3}$ : ozônio; T: temperatura; ${ }^{\circ} \mathrm{C}$ : graus Celsius; UR: umidade relativa do ar; \%: porcentagem.

Tabela 2. Analise comparativa entre o número de internações por doenças respiratórias nos períodos de queima e não queima da cana-de-açúcar.

\begin{tabular}{|c|c|c|c|c|c|c|c|}
\hline & \multirow{2}{*}{ Média } & \multirow{2}{*}{ DP } & \multirow{2}{*}{ Mínimo } & \multirow{2}{*}{ Máximo } & \multicolumn{3}{|c|}{ Percentis } \\
\hline & & & & & 25th & 50th & 75th \\
\hline \multicolumn{8}{|l|}{ Não queima (162 dias) } \\
\hline Total de internações $(\mathrm{n}=404)$ & 2,5 & 1,3 & 1,0 & 7,0 & 1,0 & 2,0 & 3,0 \\
\hline $\operatorname{DPOC}(\mathrm{n}=36)$ & 0,2 & 0,5 & 0,0 & 3,0 & 0,0 & 0,0 & 0,0 \\
\hline Asma $(\mathrm{n}=14)$ & 0,1 & 0,3 & 0,0 & 1,0 & 0,0 & 0,0 & 0,0 \\
\hline Pneumonia $(\mathrm{n}=125)$ & 0,8 & 0,8 & 0,0 & 4,0 & 0,0 & 1,0 & 1,0 \\
\hline Broncopneumonia $(\mathrm{n}=107)$ & 0,7 & 0,9 & 0,0 & 4,0 & 0,0 & 0,0 & 1,0 \\
\hline Infecções VAS $(\mathrm{n}=54)$ & 0,3 & 0,6 & 0,0 & 3,0 & 0,0 & 0,0 & 1,0 \\
\hline \multicolumn{8}{|l|}{ Queima (350 dias) } \\
\hline Total de internações $(\mathrm{n}=775)$ & $2,2^{*}$ & 1,3 & 1,0 & 8,0 & 1,0 & 2,0 & 3,0 \\
\hline $\operatorname{DPOC}(\mathrm{n}=78)$ & 0,2 & 0,5 & 0,0 & 3,0 & 0,0 & 0,0 & 0,0 \\
\hline Asma $(\mathrm{n}=21)$ & 0,1 & 0,3 & 0,0 & 2,0 & 0,0 & 0,0 & 0,0 \\
\hline Pneumonia $(\mathrm{n}=372)$ & $1,1^{* *}$ & 1,0 & 0,0 & 7,0 & 0,0 & 1,0 & 2,0 \\
\hline Broncopneumonia $(\mathrm{n}=231)$ & 0,7 & 0,9 & 0,0 & 4,0 & 0,0 & 0,0 & 1,0 \\
\hline Infecções VAS $(\mathrm{n}=141)$ & 0,4 & 0,7 & 0,0 & 4,0 & 0,0 & 0,0 & 1,0 \\
\hline
\end{tabular}

DP: desvio padrão; DPOC: doença pulmonar obstrutiva crônica; VAS: vias aéreas superiores. ${ }^{*}$ Diferente do período de não queima $\left({ }^{\star} \mathrm{p}<0,011,{ }^{* *} \mathrm{p}=0,005\right.$; Teste Mann-Whitney).

Tabela 3. Análise comparativa entre as concentrações de poluentes atmosféricos e variáveis meteorológicas nos períodos de queima e não queima da cana-de-açúcar.

\begin{tabular}{lccc}
\hline & Não queima (162 dias) & Queima (350 dias) & p-valor \\
\hline $\begin{array}{l}\text { Poluentes Atmosféricos } \\
\mathrm{MP}_{10}\end{array}$ & & \\
$\mathrm{NO}_{2}$ & $14,3(9,6-19,6)$ & $26,4(17,1-36,5)$ & $<0,0001^{*}$ \\
$\mathrm{O}_{3}$ & $11,7(7,4-15,2)$ & $16,4(12,0-22,2)$ & $<0,0001^{*}$ \\
Variáveis Meteorológicas & $45,0(35,6-53,0)$ & $47,6(37,8-61,1)$ & $0,009^{*}$ \\
$\mathrm{~T}\left({ }^{\circ} \mathrm{C}\right)$ & & \\
$\mathrm{UR}(\%)$ & $25,9(24,5-27,3)$ & $23,2(20,7-25,2)$ & $<0,0001^{*}$ \\
\hline
\end{tabular}

$\mathrm{MP}_{10}$ : material particulado de 10 micrômetros; $\mathrm{NO}_{2}$ : dióxido de nitrogênio; $\mathrm{O}_{3}$ : ozônio; $\mathrm{T}$ : temperatura; ${ }^{\circ} \mathrm{C}$ : graus Celsius; UR: umidade relativa do ar; \%: porcentagem. Diferença entre os períodos de não queima e queima $\left({ }^{*} \mathrm{p}<0,05\right.$, Teste Mann-Whitney). Dados expressos em mediana (intervalo interquartílico 25-75\%). 
sição especificamente ao $\mathrm{MP}$ tem relação com o aumento no número de internações hospitalares por pneumonia ${ }^{27-29}$.

Outro poluente emitido na queima da canade-açúcar é o $\mathrm{NO}_{2}$, este é produzido a partir da oxidação em altas temperaturas do nitrogênio do ar e responsável também por desencadear efeitos potenciais sobre a saúde respiratória como produção de sibilos, infecções respiratórias, exacerbação de asma e diminuição da capacidade pulmonar $^{30-33}$.

Mills et al. ${ }^{34}$ realizaram uma revisão sistemática sobre os efeitos agudos do $\mathrm{NO}_{2}$ sobre a mortalidade e hospitalizações, sendo identificada uma associação positiva entre a exposição e admissão hospitalar de forma aguda. Já Ghozikali et al. ${ }^{35}$ realizaram um estudo sobre os efeitos da exposição em mais poluentes como o $\mathrm{O}_{3}, \mathrm{NO}_{2}$ e dióxido de enxofre $\left(\mathrm{SO}_{2}\right)$ em pacientes com DPOC. Seus resultados mostraram um impacto significativo na internação hospitalar no inverno em comparação ao verão. Nossos achados corroboram com esses estudos, visto que o período de queima de canade-açúcar ocorre em sua grande parte no inverno e a média da temperatura manteve-se menor em comparação ao período de não queima.

Além da queima da cana-de-açúcar, a emissão de poluentes pelo tráfego veicular também é considerado um fator contribuinte para o aumento nas concentrações de $\mathrm{MP}_{10}$ e $\mathrm{NO}_{2}$. De acordo com dados fornecidos pelo site do departamento de trânsito do Município, a frota veicular também apresentou aumento no período de queima (1.810.486 carros) comparado ao período de não queima (1.265.333 carros) na cidade ${ }^{36}$.

Dentre as DR investigadas no presente estudo, a pneumonia apresentou aumento significativo no período de queima. Estes resultados estão em concordância com o estudo de Arbex et al. ${ }^{2}$, que avaliaram 1.505 internações por pneumonia, observaram aumento de 6\% (CI 95\% 2,4 a 9,9) no número de internações durante dois dias, após o aumento na concentração de partículas totais em suspensão em uma região canavieira do estado de São Paulo. No estudo de Negrisoli e Nascimento ${ }^{37}$, realizado com 1.825 internações por pneumonia em crianças, observaram que a cada $10 \mu \mathrm{g} / \mathrm{m}^{3} \mathrm{de}$ aumento nas concentrações de $\mathrm{NO}_{2}$ no mesmo dia, aumenta $16 \%$ o risco de internações hospitalares. Semelhante comportamento ocorre com o $\mathrm{MP}_{10}$, que aumenta $9 \%$ do risco de internação após quatro dias de exposição.

Outro ponto importante deste estudo foi a influência dos dados meteorológicos. Sabe-se que um dos fatores que contribuem para a dispersão dos poluentes atmosféricos é a temperatura, que em condições baixas pode provocar maior concentração de poluentes, dificultando assim a renovação do ar. O estudo de Elliot et al. ${ }^{38}$ reportaram que em dias mais frios aumentam-se o número de internações hospitalares por bronquite aguda e gripe por influenza quando comparados à dias mais quentes. Zhou et al. ${ }^{39}$ analisaram 93.538 adultos com idade acima de 20 anos e 87.748 idosos acima de 60 anos e observaram um aumento de 1.05\% (CI 95\% 0.08-2.04) na taxa de mortalidade por doenças respiratórias nos adultos e 1,62\% (CI 95\% 0,22-3,46) nos idosos durante o inverno, isso associado ao aumento mensal de $10 \mu \mathrm{g} / \mathrm{m}^{3}$ de $\mathrm{MP}_{10}$.

Deste modo, existe uma conexão entre a baixa temperatura e doenças respiratórias. A congestão nasal causada pela rinite, por exemplo, pode levar à inalação bucal e, portanto, resultando em uma resposta inflamatória ${ }^{40-42}$.

Em contrapartida, neste estudo, observou-se relação negativa do $\mathrm{O}_{3}$ sobre o número total de internações por doenças respiratórias. Entretan- 
to, as baixas temperaturas presentes no período de queima se associou positivamente com o $\mathrm{O}_{3}$. Sabe-se que a formação do $\mathrm{O}_{3}$ se dá pelas espécies de $\mathrm{NOx}\left(\mathrm{NO}_{2}+\mathrm{NO}\right)$, no entanto sofre influência do período do dia e temperatura, onde durante a noite e com a diminuição da temperatura há presença principalmente de NO, que é um importante eliminador de $\mathrm{O}_{3}^{43}$, fato que pode explicar por partes a relação negativa.

Como limitações do estudo, foram incluídas somente internações realizadas em unidades vinculadas ao Sistema Único de Saúde (SUS), excluindo as por planos de saúde privados. Também não foi identificado casos de reinternações e indivíduos residentes de outras cidades.

Diante da importância atribuída no âmbito dos agravos à saúde relacionados com os poluentes atmosféricos a partir da queima noturna da

\section{Colaboradores}

D Ramos: Concepção e projeto, análise e interpretação dos dados; Redação do artigo; Aprovação final da versão a ser publicada; Responsável por todos os aspectos do trabalho (garantia da exatidão e integridade de qualquer parte da obra). PRS Pestana: Concepção e projeto, Aprovação final da versão a ser publicada. IB Trevisan: análise e interpretação dos dados, Redação do artigo; Aprovação final da versão a ser publicada. DGD Christofaro: interpretação dos dados, Aprovação final da versão a ser publicada. GY Tacao e IC Coripio: análise e interpretação dos dados; Redação do artigo; Aprovação final da versão a ser publicada. AD Ferreira: Concepção e projeto, análise e interpretação dos dados; Redação do artigo; Aprovação final da versão a ser publicada. EMC Ramos: Redação do artigo; Aprovação final da versão a ser publicada. palha da cana-de-açúcar, o presente estudo observou maior prevalência de internações hospitalares por DR, principalmente por pneumonia durante o período de queima.

De acordo com nossas análises, estabelecemos que poluentes como o $\mathrm{MP}_{10} \mathrm{e} \mathrm{NO}_{2}$, juntamente com a temperatura, tornaram-se fatores envolvidos no processo de adoecimento por doenças respiratórias.

A partir dessas conclusões, espera-se que os resultados obtidos neste estudo sejam úteis a nível nacional, principalmente para a região centro-sul do Brasil, que atualmente é responsável por oitenta e oito por cento $(88 \%)$ da produção da cana-de-açúcar, e que ainda não possui nenhuma implantação de políticas públicas visando a diminuição desses riscos que afetam diretamente a saúde humana ${ }^{44,45}$.

\section{Agradecimentos}

Ao Instituto Nacional de Análise Integrada do Risco Ambiental (INAIRA/CNPq) e à Fundação de Amparo à Pesquisa do Estado de São Paulo (FAPESP). 


\section{Referências}

1. Arbex MA, Martins LC, Oliveira RC, Pereira LA, Ar bex FF, Cançado JE, Saldiva PH, Braga AL. Air pollution from biomass burning and asthma hospital admissions in a sugar cane plantation area in Brazil. J Epidemiol Community Health 2007; 61(5):395-400.

2. Arbex MA, Pereira LA, Carvalho-Oliveira R, Saldiva $\mathrm{PH}$, Braga AL. The effect of air pollution on pneumonia-related emergency department visits in a region of extensive sugar cane plantations: a 30-month time-series study. J Epidemiol Community Health 2014; 68(7):669-674.

3. Ito MS, Ramos EMC, Pestana PRS, Ceccato ADF, Carvalho Junior LCS, Tommaselli JTG, Proença CA, Teixeira MFS, Trevisan IB, David RM, Faustino G, Ramos D. Hospitalizações por doenças respiratórias associados à exposição de metais Tóxicos no material particulado e nível de temperatura em Presidente Prudente, SP, Brasil. Colloquium Vitae 2014; 5(2):110-118.

4. HEI Collaborative Working Group on Air Pollution, Poverty, and Health in Ho Chi Minh City, Le TG, Ngo L, Mehta S, Do VD, Thach TQ, Vu XD, Nguyen DT, Cohen A. Effects of short-term exposure to air pollution on hospital admissions of young children for acute lower respiratory infections in Ho Chi Minh City, Vietnam. Res Rep Health Eff Inst 2012; (169):572; discussion 3-83.

5. Paraiso ML, Gouveia N. Health risks due to pre-harvesting sugarcane burning in Sao Paulo State, Brazil. Rev Bras Epidemiol 2015; 18(3):691-701.

6. Riguera D, Andre PA, Zanetta DM. Sugar cane burning pollution and respiratory symptoms in schoolchildren in Monte Aprazivel, Southeastern Brazil. Rev Saude Publica 2011; 45(5):878-886.

7. Leite RCM, Guimarães EC, Lima E, Barrozo M, Tavares M. Utilização de regressão logística simples na verificação da qualidade do ar atmosférico de Uberlândia. Engenharia Sanitária e Ambiental 2011; 16(2):175-180.

8. Moraes AC, Ignotti E, Netto PA, Jacobson LS, Castro $\mathrm{H}$, Hacon SS. Wheezing in children and adolescents living next to a petrochemical plant in Rio Grande do Norte, Brazil. J Pediatr (Rio J) 2010; 86(4):337-344.

9. Nascimento LF, Francisco JB, Patto MB, Antunes AM. Environmental pollutants and stroke-related hospital admissions. Cad Saude Publica 2012; 28(7):13191324.

10. Nascimento LFC, Módolo MCC, Carvalho Júnior JA. Atmospheric pollution effects on childhood health: an environmental study in the Paraíba Valley. Revista Brasileira de Saúde Materno Infantil 2004; 4(4):367374.

11. Pestana PR, Braga AL, Ramos EM, Oliveira AF, Osadnik CR, Ferreira AD, Ramos D. Effects of air pollution caused by sugarcane burning in Western Sao Paulo on the cardiovascular system. Rev Saude Publica 2017; 51:13.

12. Paraiso MLS, Gouveia N. Riscos à saúde devido à queima prévia da palha de cana-de-açúcar no Estado de São Paulo, Brasil. Rev Bras Epidemiol 2015; 18(3):691701.
13. Silveira HCS, Schmidt-Carrijo M, Seidel EH, Scapulatempo-Neto C, Longatto-Filho A, Carvalho AL, Reis RM, Saldiva PH. Emissions generated by sugarcane burning promote genotoxicity in rural workers: a case study in Barretos, Brazil. Environ Health 2013; 12(1):87.

14. Arbex MA, Cançado JED, Pereira LAA, Braga ALF, Saldiva PHN. Queima de biomassa e efeitos sobre a saúde. Jornal Brasileiro de Pneumologia 2004; 30(2):158-175.

15. Ribeiro H, Assunção JV. Efeitos das queimadas na saúde humana. Estudos Avançados 2002; 16(44):125148.

16. Ferreira-Ceccato AD, Ramos EM, Carvalho Júnior LC, Xavier RF, Teixeira MF, Raymundo-Pereira PA, Proença CA, Toledo AC, Ramos D. Short-term effects of air pollution from biomass burning in mucociliary clearance of Brazilian sugarcane cutters. Respir Med 2011; 105(11):1766-1768.

17. Goto DM, Lanca M, Obuti CA, Galvao Barbosa CM, Nascimento Saldiva PH, Trevisan Zanetta DM, Lorenzi-Filho G, de Paula Santos U, Nakagawa NK. Effects of biomass burning on nasal mucociliary clearance and mucus properties after sugarcane harvesting. Environ Res 2011; 111(5):664-669.

18. Vormittag E, Rodrigues CG, Saldiva P. Projeção da mortalidade, internações hospitalares na rede pública e gastos públicos em saúde decorrentes da poluição atmosférica no Estado de São Paulo de 2012 a 2030. São Paulo: Instituto Saúde e Sustentabilidade; 2010.

19. Bayram H. Effects of air pollution on respiratory health. Int J Mycobacteriology 2015; 4(Supl. 1):59.

20. Instituto Brasileiro de Geografia e Estatistica (IBGE). Conheça cidades e estados do Brasil. 2017. [acessado 2017 Jul 26]. Available from: http://cidades.ibge.gov. br/xtras/perfil.php?codmun $=354140$

21. Companhia Ambiental do Estado de São Paulo (CETESB). 2017. [acessado 2017 Jul 26]. Available from: http://www.cetesb.sp.gov.br/institucional/agencias-da-cetesb/

22. Organização Mundial da Saúde (OMS). Padrões de Qualidade do Ar. 2005. [acessado 2017 Jul 26]. Available from: http://ar.cetesb.sp.gov.br/padroes-de-qualidade -do-ar/

23. Atkinson RW, Fuller GW, Anderson HR, Harrison RM, Armstrong B. Urban ambient particle metrics and health: a time-series analysis. Epidemiology 2010; 21(4):501-511.

24. Clifford RL, Jones MJ, MacIsaac JL, McEwen LM, Goodman SJ, Mostafavi S, Kobor MS, Carlsten C. Inhalation of diesel exhaust and allergen alters human bronchial epithelium DNA methylation. J Allergy Clin Immunol 2017; 139(1):112-121.

25. Hirota JA, Marchant DJ, Singhera GK, Moheimani F, Dorscheid DR, Carlsten C, Sin D, Knight D. Urban particulate matter increases human airway epithelial cell IL-1beta secretion following scratch wounding and H1N1 influenza A exposure in vitro. Exp Lung Res 2015; 41(6):353-362. 
26. Moller P, Jacobsen NR, Folkmann JK, Danielsen PH, Mikkelsen L, Hemmingsen JG, Vesterdal LK, Forchhammer L, Wallin $\mathrm{H}$, Loft S. Role of oxidative damage in toxicity of particulates. Free Radical Research 2010; 44(1):1-46.

27. Jasinski R, Pereira LAA, Braga ALF. Poluição atmosférica e internações hospitalares por doenças respiratórias em crianças e adolescentes em Cubatão, São Paulo, Brasil, entre 1997 e 2004. Cad Saude Publica 2011; 27(11):2242-2252.

28. Nascimento LF, Pereira LA, Braga AL, Modolo MC, Carvalho Júnior JA. Effects of air pollution on children's health in a city in Southeastern Brazil. Rev Saude Publica 2006; 40(1):77-82.

29. Souza LSV, Nascimento LFC. Air pollutants and hospital admission due to pneumonia in children: a time series analysis. Revista da Associação Médica Brasileira 2016; 62(2):151-156.

30. Anderson JO, Thundiyil JG, Stolbach A. Clearing the air: a review of the effects of particulate matter air pollution on human health. J Med Toxicol 2012; 8(2):166-175.

31. Gamon LF, Wille U. Oxidative Damage of Biomolecules by the Environmental Pollutants $\mathrm{NO}^{*}$ and NO3*. Accounts of Chemical Research 2016; 49(10):2136-2145.

32. Greenberg N, Carel RS, Derazne E, Bibi H, Shpriz M, Tzur D, Portnov BA. Different effects of long-term exposures to $\mathrm{SO} 2$ and $\mathrm{NO} 2$ air pollutants on asthma severity in young adults. $J$ Toxicol Environ Health $A$ 2016; 79(8):342-351.

33. Khaniabadi YO, Goudarzi G, Daryanoosh SM, Borgini A, Tittarelli A, De Marco A. Exposure to PM10, $\mathrm{NO} 2$, and $\mathrm{O} 3$ and impacts on human health. Environ Sci Pollut Res Int 2017; 24(3):2781-2789.

34. Mills IC, Atkinson RW, Kang S, Walton H, Anderson HR. Quantitative systematic review of the associations between short-term exposure to nitrogen dioxide and mortality and hospital admissions. BMJ Open 2015; 5(5):e006946.

35. Ghozikali MG, Mosaferi M, Safari GH, Jaafari J. Effect of exposure to $\mathrm{O}(3), \mathrm{NO}(2)$, and $\mathrm{SO}(2)$ on chronic obstructive pulmonary disease hospitalizations in Tabriz, Iran. Environ Sci Pollut Res Int 2015; 22(4):28172823.
36. Brasil. Departamento Nacional de Trânsito (Denatran). 2017. [cited 2017 Jul 26]. Available from: http:// www.denatran.gov.br/frota.htm

37. Negrisoli J, Nascimento LFC. Poluentes atmosféricos e internações por pneumonia em crianças. Revista Paulista de Pediatria 2013; 31(4):501-506.

38. Elliot AJ, Cross KW, Fleming DM. Acute respiratory infections and winter pressures on hospital admissions in England and Wales 1990-2005. J Public Health 2008; 30(1):91-98.

39. Zhou M, He G, Liu Y, Yin P, Li Y, Kan H, Fan M, Xue A, Fan M. The associations between ambient air pollution and adult respiratory mortality in 32 major Chinese cities, 2006-2010. Environ Res 2015; 137:278-286.

40. Cruz AA, Togias A. Upper airways reactions to cold air. Curr Allergy Asthma Rep 2008; 8(2):111-117.

41. Koskela HO. Cold air-provoked respiratory symptoms: the mechanisms and management. Int J Circumpolar Health 2007; 66(2):91-100.

42. Mourtzoukou EG, Falagas ME. Exposure to cold and respiratory tract infections. Int J Tuberc Lung Dis 2007; 11(9):938-943.

43. Sillman S. The relation between ozone, NO $\mathrm{x}$ and hydrocarbons in urban and polluted rural environments. Atmospheric Environment 1999; 33(12):18211845.

44. França DA, Longo KM, Soares Neto TG, Santos JC, Freitas SR, Rudorff BF, Cortez EV, Anselmo E, Carvalho Júnior JA. Pre-harvest sugarcane burning: determination of emission factors through laboratory measurements. Atmosphere 2012; 3(1):164-180.

45. Nicolella AC, Belluzzo W. The effect of reducing the pre-harvest burning of sugar cane on respiratory health in Brazil. Environment and Development Economics 2015; 20(1):127-140.

Artigo apresentado em 06/09/2017

Aprovado em 07/04/2018

Versão final apresentada em 09/04/2018 